\title{
With Hope and Imagination: Imaginative moral decision-making in neonatal intensive care units
}

Citation for published version (APA):

Coeckelbergh, M., \& Mesman, J. (2007). With Hope and Imagination: Imaginative moral decision-making in neonatal intensive care units. Ethical Theory and Moral Practice, 10, 3-21. https://doi.org/10.1007/s10677-006-9046-2

Document status and date:

Published: 01/01/2007

DOI:

10.1007/s10677-006-9046-2

Document Version:

Publisher's PDF, also known as Version of record

Document license:

Taverne

Please check the document version of this publication:

- A submitted manuscript is the version of the article upon submission and before peer-review. There can be important differences between the submitted version and the official published version of record.

People interested in the research are advised to contact the author for the final version of the publication, or visit the DOI to the publisher's website.

- The final author version and the galley proof are versions of the publication after peer review.

- The final published version features the final layout of the paper including the volume, issue and page numbers.

Link to publication

\footnotetext{
General rights rights.

- You may freely distribute the URL identifying the publication in the public portal. please follow below link for the End User Agreement:

www.umlib.nl/taverne-license

Take down policy

If you believe that this document breaches copyright please contact us at:

repository@maastrichtuniversity.nl

providing details and we will investigate your claim.
}

Copyright and moral rights for the publications made accessible in the public portal are retained by the authors and/or other copyright owners and it is a condition of accessing publications that users recognise and abide by the legal requirements associated with these

- Users may download and print one copy of any publication from the public portal for the purpose of private study or research.

- You may not further distribute the material or use it for any profit-making activity or commercial gain

If the publication is distributed under the terms of Article $25 \mathrm{fa}$ of the Dutch Copyright Act, indicated by the "Taverne" license above, 


\title{
With Hope and Imagination: Imaginative Moral Decision-Making in Neonatal Intensive Care Units
}

\author{
Mark Coeckelbergh $\cdot$ Jessica Mesman
}

Accepted: 5 October 2006 / Published online: 2 December 2006

(C) Springer Science + Business Media B.V. 2006

\begin{abstract}
Although the role of imagination in moral reasoning is often neglected, recent literature, mostly of pragmatist signature, points to imagination as one of its central elements. In this article we develop some of their arguments by looking at the moral role of imagination in practice, in particular the practice of neonatal intensive care. Drawing on empirical research, we analyze a decision-making process in various stages: delivery, staff meeting, and reflection afterwards. We show how imagination aids medical practitioners demarcating moral categories, tuning their actions, and exploring long-range consequences of decisions. We argue that imagination helps to bring about at least four kinds of integration in the moral decision-making process: personal integration by creating a moral self-image in moments of reflection; social integration by aiding the conciliation of the diverging perspectives of the people involved; temporal integration by facilitating the parties to transcend the present moment and connect past, present, and future; and epistemological integration by helping to combine the various forms of knowledge and experience needed to make moral decisions. Furthermore, we argue that the role of imagination in these moral decision-processes is limited in several significant ways. Rather than being a solution itself, it is merely an aid and cannot replace the decision itself. Finally, there are also limits to the practical relevance of this theoretical reflection. In the end, it is up to care professionals as reflective practitioners to re-imagine the practice of intensive care and make the right decisions with hope and imagination.
\end{abstract}

Key words imagination $\cdot$ moral theory $\cdot$ moral decision-making $\cdot \operatorname{pragmatism} \cdot$ neonatal intensive care $\cdot$ medical ethics

M. Coeckelbergh $(\triangle) \cdot J$. Mesman

Philosophy, Maastricht University, P.O. Box 616, Maastricht 6200 MD, The Netherlands

e-mail: coeckelm@gmx.net

J. Mesman

Technology \& Society Studies, Maastricht University, P.O. Box 616, Maastricht 6200 MD,

The Netherlands

e-mail: j.mesman@tss.unimaas.nl 


\section{Introduction}

The role of imagination in moral decision-making is often neglected. However, recent literature suggests that this neglect is undeserved. Against a philosophical tradition that stresses rational and principled moral reasoning, authors such as Johnson (1993), Fesmire (2003), and Nussbaum (2001) offer conceptual frameworks that highlight the moral role of imagination. However, it strikes us that the precise role of imagination in concrete moral practice remains largely undeveloped. ${ }^{1}$ This is true for both philosophical and empirical studies. ${ }^{2}$ In this paper we want to explore this role by looking at moral practice in the medical field. In particular, we will draw on research ${ }^{3}$ about neonatal intensive care units (NICUs) to show the crucial role of imagination in decision-making processes that carry a strong moral weight.

First, we will discuss some ${ }^{4}$ existing philosophical ideas on the relation between morality and imagination, in particular imagination's role in the exploration of possibilities and projection of future scenarios and in the reconstruction of the other's experience. Secondly, we will turn to decision-making practice in NICUs and bring out the moral role of imagination ${ }^{5}$ in this practice. Using a conceptual apparatus based on both philosophical and Science and Technology Studies approaches, we aim to contribute to theory on the moral role of imagination ${ }^{6}$ and to offer new insights in decision-making practices at NICUs.

\section{Literature on Morality and Imagination}

In John Dewey and Moral Imagination (2003) Steven Fesmire argues that most philosophers have overlooked the importance of the moral role of imagination. Taking his inspiration from John Dewey and more generally the tradition of American pragmatism, Fesmire objects to the

\footnotetext{
${ }^{1}$ An exception may be Werhane's book on moral imagination and management decision-making (Werhane 1999).

${ }^{2}$ Some examples of studies on moral decision-making processes in medicine are Anspach (1993), Becker and Grunwald (2000), Eliasson et al. (1997), Frohock (1986), Holm et al. (1996), and Vermeulen (2004). None of them take the role of imagination into account.

${ }^{3}$ See previous research by Jessica Mesman, in particular her study Ervaren Pioniers [Experienced Pioneers] (Mesman 2002) based on ethnographic research in NICU units in The Netherlands and the US carried out in the $1990 \mathrm{~s}$.

${ }^{4}$ To keep the scope of our paper within manageable dimensions we had to be selective, but we are aware that there is a larger body of literature relevant to the issue of moral imagination. See for example literature on moral particularism (e.g., Dancy 1993 and 2004) and narrative approaches to bioethics (e.g., Burell and Hauerwas 1977 and subsequent work in this domain by Brody, Hunter, and Nelson.) Furthermore, without relying on the pragmatist, particularist, or narrative traditions in ethical theory Bernard Williams has recognised in his Ethics and the Limits of Philosophy that "ethical understanding needs a dimension of social explanation" and was sympathetic to a view of the ethical life as social practices that can change (Williams 1985: 131).

5 The focus on the moral role of imagination in this paper is part of a larger ongoing research project by Mark Coeckelbergh.

${ }^{6}$ We are aware that there is a question about the precise meaning of imagination, but a full discussion of this problem is not within the aims and scope of this essay. Here we will focus on the question regarding its role, and follow the literature referred to above: imagination often appears to refer to projection: projection of future scenarios and empathic projection. Do they have this meaning in common? Is it a matter of family resemblance? We suggest further thinking to work out if there is a 'central' or underlying meaning of imagination as used in this paper.
} 
view that principles, rules, and procedures have a larger role than "guiding hypotheses that help open situations to inquiry" (Fesmire 2003, p. 59). He claims that when a problem is encountered, alternatives "are tested in thought as we imaginatively envision them carried out" until a solution is found (p. 35). Rather than reason, he argues, we need imagination as empathetic projection and creatively tapping a situation's possibility. Imagination allows us to enter in the situation of others, and to "concretely perceive what is before us in the light of what should be" (p. 65). In this way perception is amplified beyond our immediate environment. Only then moral judgment and moral deliberation is possible.

Fesmire uses the term "dramatic rehearsal" to stress the social character of deliberation: "to deliberate is to co-author a dramatic story with environing conditions in community with others" (p. 78). Furthermore, he makes a comparison between morality and jazz improvisation: to play together we must harmonize interests and respond to each other's signals to arrive at a common result. "Drawing on the resources of tradition, memory, and long exercise, she [the jazz improvisator] plays into the past tone to discover the possibilities for future tones in the way moral imagination enables us to see the old in terms of the possible" (p. 94).

Fesmire is not the first to point to the central role of imagination in moral reasoning. For example, in Moral Imagination (1993) Mark Johnson has argued that moral understanding and deliberation depend crucially on the cultivation of imagination and not on the deduction of principles. He shows that the metaphors we use embody a shared moral understanding that grows out of experience. He uses evidence from cognitive science and linguistics to show how we use metaphors to understand situations, and how our conceptual systems are grounded in bodily experience. Morality, according to Johnson, is not a system of laws but an imaginative exercise based on what we share with others. Moral principles and rules still have a role to play - they encapsulate our past experience - but we should engage in open dialogue about them.

Furthermore, Martha Nussbaum is known for her view that literature is to stimulate empathy (or sympathy), which she defines in Upheavals of Thought (2001) as the "imaginative reconstruction of the experience of the sufferer" (Nussbaum 2001, p. 327). To imagine the sufferer's experience does not mean we become the sufferer, she argues, but rather than we imagine what it is like to be in the sufferer's place while being aware that we are not the sufferer. Furthermore, by appealing to the example of the empathetic torturer she makes a distinction between empathy and compassion: someone might exercise empathy while not feeling compassion at all or even enjoying the suffering of the other.

In spite of these interesting theoretical contributions to thinking about the relation between imagination and morality, however, little work has been done on how imaginative reasoning works in practice. What is its precise role in moral reasoning in general, and decision-making in particular? What exactly is the relation between general rules and imagination? To explore these questions we now turn to moral decision-making in the concrete practice of neonatal intensive care.

\section{Imagination and Neonatal Intensive Care}

\subsection{Introduction: Decision-making in Neonatal Intensive Care Units}

Neonatal intensive care units specialise in the care and treatment of newborns. If these extremely fragile children end up in the NICU, it means that their lives are seriously at risk. Furthermore, NICU staff members generally have little time for taking decisions, and rapidly changing technology that can potentially do more harm than good adds further 
medial and moral difficulties to the decision-making process. In such a context, moral dilemmas are the rule rather than the exception. For example, to decide to discontinue the child's treatment may rob it of its last change of discovery, but to continue treatment may only contribute to more suffering with little chance of improving the child's medical condition and quality of life.

The standard answer to such decision-making problems is regulation. Protocols are designed to guide and direct the staff's decision-making. But the problems these protocols start off from are seldom identical to the problem at hand. Rather, staff members are challenged to resolve the gap between general moral categories with the child's unique situation. We will argue below that in this process imagination plays a crucial role and can only take place in a space of freedom not colonised by regulation ${ }^{7}$. Using real life stories we will explore a variety of ways in which imagination plays a role in making moral decisions. We will consider not only the staff but also the parents. We structured our discussion chronologically, by looking at three phases that involve moral decision-making: decisions made in the delivery room, decisions made in a staff meeting, and reflection on these decisions afterwards by those involved. Let us start in the delivery room with the story of Esther.

\subsection{In the Delivery Room: Projection and Empathy (Phase 1)}

\subsubsection{Introduction}

A woman is admitted to the delivery room while she is only 6 months pregnant. She expects her first child. But a severe toxaemia of pregnancy endangers the situation of the mother. Immediate action is required to save her live. There are two options available: the use of medication or a caesarean delivery that stops the pregnancy. In both ways the mother will be cured, but the implications for her baby differ very much. The first option (medication) implies the baby will die an intra-uterine death. The second option (caesarean) means that after the delivery the baby will be taken to the neonatal intensive care unit (NICU) and will receive maximal support. In other words, the treatment of the baby (a non-treatment policy or maximal intervention) and that of the mother (medication or caesarean) are intertwined. Now it is up to the parents what to do. The child is in distress and a CTG shows its diminishing heart rate. The risk of losing the child increases. The necessity of getting the baby out and to opt for the caesarean delivery seems obvious. But the condition of the baby in the womb is such that it has taken away the self-evidence of medical intervention. The baby is just 25 weeks of gestational age and it is even too small for its age. Given these facts there is a chance that the baby will not survive and if it does chances are high it will become severely handicapped. The alarm of the CTG shows how the heart rate of the baby drops again. Something needs to be done. The doctors ask the parents whether or not to intervene. Since an explicit choice is asked for let us look at the moral reasoning involved in this case, at how imagination plays a role in the choice between treatment and nontreatment.

\footnotetext{
${ }^{7}$ For a discussion of the advantages and disadvantages of regulation see also Coeckelbergh (2006) and Mesman (2002). 


\subsubsection{Projection}

A decision about treatment or non-treatment depends much on the expectations doctors have about the future of the child. It is difficult to foresee that future here since there is little information available. The neonatologist informs the parents about the condition of their baby:

We have to realize that this baby is only 25 weeks of gestation and it has the additional disadvantages of being too small for its age. We have to take into account the probability of severe handicaps.

In pointing out the consequences of a choice for treatment he projects the possible future for the baby. But the doctors remain uncertain about what to do; their projection is not sufficient for taking a decision. The parents have to decide in what way the doctors need to intervene. Therefore, the father wants to know the exact figures. The neonatologist formulates his words very carefully:

It's very hard to predict these kinds of things. I can only give you a rough estimation that she has a $10 \%$ chance that she will be ok, an $80 \%$ chance that she will survive but will be severely handicapped and a $10 \%$ chance that she will not survive. But again I have to stress the fact that these figures are just a rough estimation.

"So, there is a chance?," the father replies. The neonatologist nods and refers to the possibility to stop treatment in a later stage. Thus, a treatment trajectory is projected, as well as its possibility to be stopped. Furthermore, different future scenarios of the child are projected: death, handicap, or relative health. The father argues:

"So, it is a choice between 'taking a risk and giving it a chance' or 'avoid any risk and take away every chance. I think that in spite of her slim chances we have to give her the benefit of the doubt and go for it. But I don't want her to live a life as a severely handicapped human being. Not for my own sake but for her. But if she has a chance, no matter how small, I think we should go for it."

On basis of the statistical figures the father opts for a caesarean delivery - implying maximal intervention after birth. The mother too uses projection to take her decision. But she does not see the child's future, but her own very nearby future. This is her description of what happened in the delivery room:

At some point the heart beat slowed down and at times even disappeared completely.

You can't imagine how it is to hear how the heartbeat of your child stops suddenly [...]. So something had to be done quickly. I didn't want the child to die in my belly.

To have a dead child in my belly was such an unbearable idea. It had to go out.

Thus, the mother had a very different image of the situation than the father or the neonatologist. She did not hear figures or percentages of survival after the child is born, but the alarm of the heart rate monitor indicating the present condition of the unborn child in her womb. She defines the situation in other terms by imagining that the child could die every moment in her womb. The imagination of the mother is focused on her own condition in the presence. As such her projection does not exceed the foetal stage of her child (we will return to this below).

These observations can now be further interpreted in terms of the moral role of imagination. We have seen that all parties involved (the neonatologists, the father, and the 
mother) are involved in a process of prognostication in which they project future scenarios to test the implications of their decision. They use their imagination to see what would happen if they decide for treatment or non-treatment. The father imagines a child with a chance of having a reasonably good life, the mother imagines a dead child in her womb. Decisions about intervention are taken on the basis of a prognosis. The actors involved all deploy experiences from the (recent) past to interpret both the developments that occur along the trajectory and - by extrapolation and imaginative projection -the ones that are likely to occur in the near future. This gives rise to specific "horizons of expectation" (Koselleck, 1985), whereby the extent to which expectations are realized plays a palpable role as well. In their formulation of new expectations, the parents and doctors rely on subsequent knowledge. This may cause them to adjust their expectations in a specific direction. Thus prognostic processes involve a linking of past (experience) and future (expectations) in the present, resulting in a "current past" and a "present future." The imagined scenarios concern themselves and/or the child, the projected images are typically linked with emotions and values the projection uses the information available about the present condition of the child, and the imaginative move helps to take decisions under conditions of time frame collapse and uncertainty. Let us explain what we mean.

First, the parties involve may imagine the child, but also themselves. For example, a nurse may imagine how it would be for her to care for a particular child for another 3 weeks. And parents may imagine how it would be for them to care for a handicapped child all their life.

Secondly, emotions such as fear, doubt and hope are related to each projection. All parties involved experience doubt and related emotions, the father fears a handicapped child, and the mother fears a dead child in her womb.

Thirdly, to overcome uncertainty imaginative reasoning is used. Both the father and the doctor used statistics to decide that she still had a chance to survive. And there is the heart rate and the alarm signals produced by the CTG machine. But the information is limited. Imagination is required to fill this gap. Imagination projects the implications of decisions. But imaginative reasoning is constrained by what we may call the sources of imagination. In other words: imaginative reasoning differs from fantasizing in that the first is bound by situational aspects as in this case statistics, numbers, and appearance.

Note that there may be competing images and thus competing scenarios for interventions. The use of imagination is different for different roles: since access to various sources varies, the results of imagination vary as well. For example, doctors have other information available than parents, and may project a different image. The use of imagination also varies on the time horizon. Esther's mother, carrying the child in her womb, could only imagine the very near future (the image of the dead child in her womb) whereas the father imagined a time after birth with the child having a chance of recovery. People have different spaces of experience and horizons of expectation and imagination can either move within such a horizon or space, or allow us to extend its borders (enlarging the horizon), sometimes opening up new horizons of experience and action.

Fourthly, when the decision-process gets stuck and immobilizes the therapeutic process, imagination helps to get it going again. For example due to time frame collapse, meaning there is suddenly little time to decide. Typically, people stand under moral pressure not to let this situation continue for too long: they often have to decide quickly. Imagination also helps to come with uncertainly: there is a knowledge gap, and this gap is filled with projected images. The epistemological status of this knowledge is not as 'hard' as some may wish, but it still supports the decision. To conclude, under conditions of immobility, whether it is due to time frame collapse or uncertainty, imagination helps people to create room for action. 
To conclude, imagination is a crucial element in the decision making process in the delivery room and the moral reasoning involved in it. This imagination is anchored in knowledge and experience; it is not as free-floating as some may think. In the present use of imagination as the projection of scenarios, the past becomes current and is connected to the future that becomes present in a way that is constrained by the available knowledge and experience of the situation of the child, its case and that of others it is compared to. ${ }^{8}$

Projecting is not limited to the delivery room. In the Neonatal Intensive Care Unit prognosis plays a dominant role as well: here too projecting is a form of imagination that supports the staff and parents in the process of prognostication. There are different ways of projecting. A distinction can be made between two structures: the cyclical repertoire and the linear repertoire. In a cyclical repertoire of projecting the course of events is conceived as following a steady pattern. Present, past and future are part of one and the same temporal system. They each function as a fixed moment in a natural cycle in which the past, for instance, is also the "future present." This cyclical structure renders the future both predictable and inevitable. Consider statements about Tom. When Tom was born prematurely at 25 weeks of term, weighing less than one and a half pound upon his admission to the NICU in a Dutch Medical Centre. In the first weeks already there were signs that Tom did not respond well to the treatment; his tendency was to get off course. His lungs did not begin to function properly on their own. And worse, Tom was developing a chronic lung insufficiency that was caused by tissue damage due to prolonged artificial respiration. One day, Tom was referred to as "a second Mick." In this remark the past functions as a mirror that reflects lessons for the future. In this cyclical form of projecting, a child's prognosis is logically deduced from past situations: "We have had children like him before. You remember Mick. At one point you know how things stand." The reference to Mick implies the message "this is how it ended before and how it will happen again. Let this be a warning!" In a linear repertoire another conceptualization of time in projecting prevails. Arguments that exclusively point to the past are rare. A linear repertoire is more open, meaning that what lies ahead is not dictated by some natural order. This kind of projecting basically comes in two versions: the future is seen as entirely open-ended, or, in a more moderate version, the future is seen as tied to probability. For example, a posteriori the attending physician articulates Tom's prognosis in terms of chances: "Theoretically, Tom still had a chance to get off the respirator. But as the days went by this chance would grow smaller." In this moderate version the various options that are looming behind the horizon of expectation can be arranged by probability (cf. Koselleck, 1985). The future is neither given nor entirely open. The number of possibilities, however, is finite and the likelihood of their occurrence is still tied to past experience. The reference to "chances" also implies that the past can repeat itself and that thus it renders the future predictable. But in a linear repertoire the future is never entirely dictated by the past. Surely, this may give rise to uncertainty, but it also leaves room for the view that the future can be construed - that history is a product of human intervention. The temporal structure of this historical awareness implies progression. When reasoning from a linear form of projecting, one is not

\footnotetext{
${ }^{8}$ In terms of rhetoric, an analytical difference can be made between two styles of reasoning: a 'case repertoire' and a 'situation' repertoire. The former involves reasoning that talks of the child as a medical case (a case of ...), making comparisons with other cases: the available medical evidence as reported in the literature, statistics, etc. The latter involves reasoning that takes the particular situation of the child in question as its starting point.
} 
concerned with individual moments in a repetitive whole, but with conceptualizing past, present and future as subsequent stages in a development marked by progression. In this form of projecting, for instance, statistical data always have a provisional character rather than a lasting validity. New knowledge and experience will increase children's chances of recovery in the future. Therefore, in the linear repertoire of projecting the future holds the promise of recovery. It is this promise to which Tom's father is hanging on.

\subsubsection{Empathy}

Projecting future scenarios is not the only thing imagination can do for us when we are involved in moral reasoning and decision-making. It also turns out to be central once we consider the role of empathy. Empathy means to put yourself in the shoes of another. Consider again the reasoning of Esther's father. He wanted to be able to stop treatment, because he did not "... want her to live a life as a severely handicapped human being." This involves an imaginative move on the part of the father to put himself in the shoes of his child when living its (future) life.

The medical staff uses empathy as well. Let us consider the case of Tom again. In the months that follow additional problems present themselves. As Tom grows larger, his agility increases. To prevent him from accidentally removing his oxygen tube, he is given sedatives. The result is addiction and a stomach ulcer. But there are other complications as well. Given this long list of problems it is no wonder that as many as four times he entirely collapsed, his heart beat going down without automatic correction. At the explicit request of his parents, the doctors do everything to save him and each time successfully so. What they do not succeed in, however, is to get him off the respirator. Consequently, after 4 months Tom is still in the NICU. While other infants are dismissed after a few weeks and allowed to go home, his recovery seems a distant possibility at best. Tom's condition is serious indeed and as his problems have increased, it has even become an issue whether or not they should go on treating him. Some nurses view Tom as a child whose suffering is no longer acceptable. They feel this suffering is caused by the physicians involved, who do not know "when to stop" and who go on treating the infant largely because the various technologies at their disposal allow them to do so. The doctor in attendance also considers Tom a child with a bad prognosis, but he refers to research of children in a similar situation that clearly suggests there is still a chance of Tom pulling through. This is why he feels that stopping with treatment is no real option yet.

In this case nurses wanted to stop treatment earlier than the doctors, and this may be explained as a difference in experience and in the role of empathy. Nurses, being in frequent contact with the child, have more direct experience of the suffering of the child. They imagined how it would be to be in the child's place. This made them decide, earlier than the doctors, that further treatment was no longer desirable. Like projection, also this form of imagination, is restricted by circumstantial factors. The following is an example of a nurse whose empathy is considered as beyond professional limits.

Beth, a nurse in an American NICU, has been in charge of caring for Dave, a premature baby that has been in the NICU for 6 months already and fully depends on artificial respiration. Like his parents, Beth believes that 1 day Dave will be able to go home. Although her fellow-nurses do not share this prognosis, they understand her position, for they know that caring for a chronic child frequently leads to a high level of attachment. The members of the nursing staff are aware of how such processes work and they know how to support Beth. As one of them says: 
It is obvious that Beth is denying the hopeless situation of Dave. But we have all been in charge of caring for a chronic child and we know what it means. You do not see how attached you have grown to a child when you are still fully engaged in providing care to it. Only when it is too late you realize how much you bonded. It is as if the child is your own baby. [...] Now Beth is in such a situation. Her life is now turning around Dave. We have tried to make her keep more of a distance by having her care for another child. But this is precisely what she does not want to do, for only she can care well for Dave, she feels.

Beth was said by her colleagues to be on the point of collapsing. For a care professional, it seems appropriate to limit empathy at some point, or not to let empathy become identification, ${ }^{9}$ or to remain in control of emotions resulting from empathy (such as compassion $)^{10}$ and not let them hinder further action (care).

Note that suffering itself need not be experienced as meaningless by the parties involved. In particular, if the medical staff and the parents are able to make sense of the child's suffering by seeing it as a (temporary) part of a story of recovery they project with the help of their experience and their imagination, this particular suffering is seen as meaningful and medically functional. If one of the parties does not see the particular suffering in this way, however, she will take a different view. This was the case with Tom, when the nurses understood continuation of treatment as meaningless. And here too, imagination played a key role: the story projected is one of endless suffering, and empathy allowed to qualify such scenario as morally undesirable.

\subsection{The Staff Meeting: Demarcation, Categorization, Alignment, and Improvisation (Phase 2)}

\subsubsection{Introduction}

Let us return to the story of Esther. After she is born, she is admitted to the neonatology ward. On the first day, an ultrasound of Esther's head shows a fourth-degree brain haemorrhage on the left side. The next day a second ultrasound shows a spreading of the bleeding to the right half of the brain. In the afternoon the child is not doing well at all, her condition gets worse. The attending physician once more looks at all the lab results and draws his conclusion: "This is not going anywhere, we'd better stop. I propose that this afternoon we have a meeting on this case in the staff room at half past one." Now the staff faces the morally difficult task to decide about whether or not to discontinue treatment.

\footnotetext{
${ }^{9}$ There is an important difference between empathy and imagination. As Nussbaum has argued, when exercising empathy we retain the awareness that we ourselves are not the sufferer, therefore there is always a kind of 'twofold attention' (Nussbaum 2001, p. 327).

${ }^{10}$ Note that empathy and compassion are not necessarily linked. As Esther's story shows, other emotions, such as hope and fear, may accompany or result from empathy as well. Furthermore, empathy may not be followed by compassion at all. There is controversy about the moral neutrality of empathy itself. Some hold the view that empathy itself is an emotion, one with a morally good connotation, and that imagination helps to imagine yourself in someone else's shoes. Others do not make this distinction and understand empathy as a form or function of imagination itself, empathy then being itself morally neutral. Appealing to the figure of the empathetic torturer, Nussbaum (2001) has argued for a distinction between empathy and compassion, although she does qualify her view a little by claiming that empathy always at least involves the recognition of the other's reality and humanity.
} 


\subsubsection{The General and the Particular}

In the context of intensive care practice general rules are typically embodied in protocols. But here we study cases and decisions that count as belonging to a 'grey area,' defined precisely as an area where conventional guidelines and protocols do not apply (easily). In such cases, as we have shown, imagination plays a central role in decision-making. Does this mean rules do no longer matter? What is the precise relation between general rules and imagination? Let us take up again the issue decision-making about (dis) continuation of intervention. Setting the boundary beyond which intervention is to be discontinued is not an issue that can be simple resolved by making up a general rule or a general category. The morally and medically hard question is, of course, whether or not the child belongs to this category. It seems to us that to make it possible to answer this question, the use of imagination as discussed above is crucial. Let us take a look at the staff meeting about Esther's condition to analyze the role of imagination in moral reasoning. Doctors at her bedside argue that even if she survives, she will be severely handicapped, and they suggest refraining from further treatment.

The intern opens the meeting with a report on the course of Esther's condition since her birth, ending with her current condition. She concludes her introduction with the question: "What will be our policy, given the current problems and taking into account the child's immediate future and its very bad prognosis?"

"What is "very bad'?", the social worker asks the intern.

The intern replies: "A fourth-degree bleeding at both sides of the head makes her chance of survival very small and if she survives at all, what kind of life are we talking about?"

In the Academic Medical Centre there is a general rule that if there is a reasonable chance that the child will survive but will suffer due to severely handicaps, medical intervention should be withhold or withdrawn. ${ }^{11}$ This consensus seems to suggest an unproblematic setting. However, the hard question is as to whether Esther falls into this category. The rhetorical question of the intern "what kind of life are we talking about?" suggests she does. Imagining Esther's future on basis of the information available tells him to stop treatment. Both the intern and the attending neonatologist have categorized Esther as a child whose condition and prognosis are that bad that it no longer legitimizes continuation of treatment. This could have been the end of the meeting. However, two neonatologists who are not involved directly in Esther's treatment join the discussion and one of them takes on the role of devil's advocate. He does so not because he mistrusts his colleagues' intentions. Rather, by taking up this role he does exactly what he is expected to do in this setting, namely to put pressure on the proposal so that its potentially weak spots can be traced. In this way, a certain level of detachment is created that offers room for addressing other facets of the problem. He asks for an EEG in addition to the ultrasound, and refers to medical literature to argue that there is still a chance.

One of the neonatologists: "Has an EEG been made as well?"

The intern: "No EEG was made. If you think it is useful for determining our course of action, we should have one made. But what are the criteria you use when the results come in?"

\footnotetext{
${ }^{11}$ Unless the parents opt for the continuation of a treatment. They take the final decision. 
The neonatologist: "I believe that with a normal EEG you cannot make a life-ending decision."

"Do you really think," the intern asks, "that with this cerebral condition there is a chance of getting a normal EEG? Do not get the wrong idea; I only try to explore the boundaries of the new parameters on which to base our policy."

The other neonatologist supports his colleague's proposal: "I think that having an EEG made is useful. What we are faced with here is to try to determine this child's prognosis together. That is difficult if you only look at the ultrasound. I wonder whether on the basis of an ultrasound we are allowed to take such a decision. The literature shows that a fourth-degree bleeding itself is not conclusive. The exact location and spread also play a role. Reference is made to a study of twenty children with a fourth-degree bleeding of which only one is moderately handicapped. I doubt, therefore, whether the currently available data allow us to start up a discontinuation of treatment."

These manoeuvres show that categorization involves something else than just a simple application of a general rule to a particular case. Moreover, the boundaries of the category itself are topic of debate. At the bedside a huge haemorrhage on both sides of the head acted as a 'moral turning point.' A moral turning point involves a medical fact that establishes the difference between liveable and unliveable life and demarcates the boundary between meaningful and meaningless treatment. However, that afternoon in the staff meeting, the boundaries that determine what does or does not belong to the category 'untreatable' or 'hopeless' have been questioned by the 'oppositional' neonatologist. For him, merely certainty about the presence of a fourth-degree brain haemorrhage is not enough: he believes that its location in the brain is equally relevant. The child, he claims, is in the category 'untreatable' if it has a brain haemorrhage of a certain size and in a specific location. Correct information on these two aspects may cause this neonatologist to believe that a moral turning point has been reached. Therefore he stresses the involvement of a radiologist.

"It is more careful to have an expert look at it," the neonatologist argues once again.

The attending disagrees: "I feel that meanwhile we are also quite capable of interpreting ultrasound pictures. We, who have been doing this for years, have more experience than the average radiologist who does it for us. Suppose we hadn't seen that bleeding on the ultrasound and we only had the clinical picture of the child being hypotensive. Then right now we would all be very worried, knowing that from a prognostic angle it is a bad sign. In prognostic terms we have various criteria that together with the relatively objectively given bleedings point into the wrong direction. There is the previous history and this child is currently in a stable bad condition. This is why I would be willing to go along with the advice of refraining from further treatment in this child's case, which from the very beginning had a very bad prognosis - or, at least, to advice as much to its parents."

The attending doctor does not need the advice of radiologist. Moreover, he even suggests that he does not even need the proof of a brain haemorrhage to categorize Esther's future as unliveable. He imagines another past - a past without the detection of the haemorrhage - to stress the bad prognosis of this child. By doing this he tries to diminish the importance of the bleeding and as such the need of additional prognostic evidence. Unlike his colleague, the attending does not raise the issue of 'such cases in general.' $\mathrm{He}$ has to deal with other factors, such as lack of time. When the neonatologist proposes the need for an elaborate written report of a radiologist, the attending reacts surprised: 
"But that would take at least two weeks and we don't have so much time. In this case, it seems to me, a corroboration of the x-ray by one of the staff members will do as well."

In this process of demarcation the boundaries of the moral category shift back and forward. Now the moral turning point has shifted back and forth from 'the current clinical picture of the child' to 'a fourth-degree bleeding at both sides of the head' into 'a fourthdegree bleeding at both sides at a specific location.' Imagination allowed the medical staff to project future and past scenarios. Both a possible past (one without the detection of the haemorrhage) and a possible future played a role in their argumentation. It gave them the opportunity to harmonize the conditions of the general categories involved and the specificity of the situation. It helped them to define the specific coordinates of the moral turning point on Esther's treatment trajectory.

\subsubsection{Drama and Improvisation: The Social Aspect of Imagination}

Decision-making in a NICU always takes place in a social context. The decision is the outcome of a process that cannot be reduced to one moment and one person. Sometimes this communal aspect is upfront: the medical staff at the Academic Medical Centre wants the advice that has to be communicated to the parents to be a decision of the medical team. In Fesmire's terms, 'harmonising' and 'tuning' is needed to achieve this consensus. To reach this consensus other prognostic evidence, such as an EEG, is required, as well as other procedures and standards. Only in this case, the whole staff (including the two neonatologists) will agree, a discontinuation policy should be started. In this process of harmonizing and fine-tuning improvisation plays an important role.

But decision-making involves not only many people but also many factors, and the moral reasoning involved in these processes consists of so many building blocks, that to be able to make a decision we need to put these factors and blocks into a coherent picture. Our point about the role of rules, standards, and protocols in moral reasoning can be stated in these terms as well: they are part of the picture, but they need to be aligned with the particularities of context, situation - here the concrete situation of the child, the culture of the unit, etc. To accomplish this contextualization those involved had to align the different elements involved. Therefore, next to categorization, alignment of the different elements involved is needed. In what way does imagination plays a role in what we may call 'fine tuning'?

The discussion described above, shows how a general concept like 'unliveable' has been concretized into medical facts (fourth-degree haemorrhage of specific size and at specific location in the brain) that require specific prognostic evidence, like an expert report of a radiologist. This piece of prognostic evidence is opposed by the attending doctor due to a lack of time. Given the lack of time, improvisation is needed. In order to prevent the negotiation process from stalling the attending searches for a formulation that would meet both positions. Improvisation helps him to address his colleague's request and at the same time reformulate it in terms of his own position. The neonatologist too is looking to find a course in-between as a way of convincing his colleague, without discarding his own conditions.

The neonatologist: "Only, that is, if we are not mistaken in our interpretation of the ultrasound. Is it an idea to ask Robert [paediatric neurologist] once again, and find out whether he has some thoughts on the matter? The only thing is that he cannot examine the child because it is being administered sedatives. But he may have a look at the ultrasound and give advice about an EEG's level of information and whether or not it is sufficient." 
A new action option is improvised to meet the challenging conditions at hand. To move beyond the impasse both the attending and the neonatologist actively undertake an effort to reconcile the two proposals with each other. In this process of harmonizing the neonatologist modifies the general standard of having an EEG examination by agreeing to only a consult by a neurologist. The standard that an extensive report from the radiologist is necessary is also adapted to the circumstances of the situation. It is agreed that consenting to the existing report suffices. Thus the neonatologist accommodates the specificity that Esther's situation calls for. The attending, in turn, reformulates his proposal in such way that it incorporates his colleague's conditions sufficiently:

Let's agree that one of the staff members from radiology looks at the ultrasound and that the child neurologist is being informed of this case with the question whether he has something to add to it. Then we can go to the parents.

The intern summarizes the decision: "If the radiologist arrives at the same conclusion as we did and the child neurologist describes the same picture as we did, our advice will be to refrain from further treatment?" All nod in agreement. The meeting is closed.

The social aspect of decision-making, moral reasoning, and imagination also implies that the moral burden or moral cost (Williams 1981) is not exclusively carried by the individual, but shared by those involved in the morally difficult decision. In the NICU context, care is taken to take the final decision in the context of the staff meetings, and communicate that decision to the parents as such: as a collective decision. Although a first decision may be taken by a doctor when standing near the child, the final decision is 'hardened,' made more robust, in the staff meeting. Furthermore, respecting the parents' autonomy, this decision is not the last word. The view of the parents is not only taken into account already in the staff decision, they also are given a say afterwards. For example, Esther's parents have to make an explicit statement about their decision to refrain from further treatment. Thus, the final decision is a collective one, and the end-result of a process of re-location (from incubator to staff room to parents room). This way of making decisions may be particular to the European, or even Dutch cultural context, with its stress on overleg (a Dutch term which may be defined as communal decision-making aimed at consensus), but the point is here that this also means that responsibility for the decision is shared, and that improvisation as a form of imagination is an essential part of the process of harmonizing in which such a shared decision is created. Let us look again at the discussion:

The intern: "If the results of the radiologist correspond to ours and the child neurologist paints the same picture as we have done, our advice is to refrain from further treatment?"

The attending: "Yeah, if at least we all can agree?"

The neonatologist: "I'm not too comfortable with the term 'advice'. I think you have to be clear to the parents about the very bad prognosis."

The social worker tries to understand the neonatologist's aversion. "But Ruud, you claim not to be very happy with the term "advice". Do you mean that you want to see the parents decide after all?"

Yes, in part I do. Even if we will not put it that way. Of course we will discuss it with them in more subtle terms than we are doing right now. But I feel that at least to some extent they should decide. 
The attending: "I fundamentally disagree with you. If you give them the impression that it is their decision, we put an enormous weight onto their shoulders. There will be the risk that whatever choice they make they will always feel guilty. I believe we should give them a compelling advice, that is, basically take the decision for them by telling them that there is really nothing we can do anymore. Thus the parents will not have to carry that burden."

(...) "Let's agree that one of the staff members from radiology looks at the ultrasound and that the paediatric neurologist is being informed of this case with the question whether he has something to add to it. Then we can go to the parents."

The intern summarizes the decision: "If the radiologist arrives at the same conclusion as we did and the paediatric neurologist describes the same picture as we did, our advice will be to refrain from further treatment?" All nod in agreement. The meeting is closed.

In the context of NICU, moral decisions do not involve the application of general ethical rules to a unique situation, but call for another activity. Significantly, in the NICU practice morality has to be custom-made, tailored to size. In fact, morality mainly appears to involve the fixing of a moral turning point: the boundary that cannot be crossed. Here morality means demarcation. This demarcation is construed on the basis of a range of divergent activities by several actors in various sites and at different moments. Custom-made morality requires testing the robustness of prognostic facts and the elasticity of moral categories, exploring the room for action, searching the right words, creating a sense of shared concern, exploring disciplinary boundaries and distributing responsibility, and learning to deal with the consequences of the decision. We have shown that in this set of activities imagination does play a role. In sum: imagination is necessary in the process of harmonizing between the general and the particular; between the staff members involved; between the different prognostic elements

After the meeting, both the radiologist and the neurologist confirm the bad prognosis of Esther. As agreed, the attending communicates to Esther's parents that the medical staff considers discontinuation of treatment appropriate in Esther's case. On basis of their parental authority the parents have the last say. They too agree to it. Esther is disconnected from the respirator and dies. Next, she is freed from all tubes and threads. The monitor screen goes blank.

\subsection{Reflection Afterwards (Phase 3)}

In contrast to what the literature sometimes may suggest to the inattentive reader, using your imagination is not itself a solution to moral problems. The medical staff had to rely on evidence that did not clearly point to one decision. Often, even if a decision is taken, doubt and uncertainty remain, and imagination was put to work again to project how things could (and should?) have been done differently. Esther died $2 \mathrm{~h}$ after the formal decision was taken to stop her treatment. Her death was caused by the fact that she was disconnected from all vital technological support. Not in all cases children die immediately after the decision is taken to stop treatment. Tom, for instance, stayed alive for another 5 weeks after the decision was taken to stop active treatment.

When Tom was born, no one knew at that time that this was the beginning of a monthlong process of waiting and hoping, of Tom suddenly collapsing several times and gradually regaining strength again. In the first weeks already there were signs that Tom had was developing a chronic lung dysfunction. In the next months his respiratory failure was 
followed by additional problems like stomach ulcer, infections, brain haemorrhage, presented themselves. Given this long list of problems it was no wonder that as many as four times he entirely collapsed, meaning his heart beat going down without automatic correction. At the explicit request of his parents, the doctors did everything to save him and each time successfully so. What they did not succeed in, however, was to get him off the respirator. Considering his bad condition and the fact that he kept resisting his oxygen tube the staff decide to stop treatment. His parents agreed. Unlike Esther, Tom was not taken off the respirator. In case of an unintended extubation they would not re-intubate the oxygen tube again, nor resuscitate him when needed. Since they imagined a trajectory of suffering, they opted for a DNR-policy (do not resuscitate) which implies that eventually the child will die by itself (by its own action). Apparently both staff and parents drew a moral distinction between active intervention (take the baby off the respirator) and passive nonintervention (do not reintubate when accidentally extubated). The latter option was preferred; no-one wanted to cross this passive/active boundary.

Although the decision has been taken to stop Tom's treatment, this does not imply the end of moral reflection. Doubt, uncertainty, hope, and despair continue. In this section we discuss the moral problems that might arise after a decision has been taken to discontinue treatment. Imagination, it will turn, plays a key role in post-decision moral reflection. The conditions for such reflection differ from pre-decision reasoning (deliberation) since there is (more) time to think.

\subsubsection{Competing Scenarios}

After a change of policy, Tom was only taken care of and no longer object of daily diagnostic tests and examination. As a result Tom's condition seemed to improve. Signs of improvement, like less agitation, more sleeping hours and increasing food intake, lead to the imaginative construction of new scenarios - competing scenarios. Encouraged by the (seemingly) better condition of Tom, the parents requested for an update of his condition to which the doctors agreed. Because of these diagnostic tests, the nursing staff became confused about the treatment policy. Did these diagnostic tests also imply a re-intubation in the case Tom would be able to throw out his oxygen tube? Some nurses actually shared the hope of the parents. They too started to doubt whether the decision to stop his treatment and apply a non-resuscitation policy was the right one. The doctors, by contrast, judged Tom's improvement to be one in appearance only. The x-rays of Tom's lungs were a clear example of the devastating effect of the respirator. This effect was in Tom's case irreversible. Based on this knowledge, they projected a scenario of temporary improvement of his general condition followed by death. These two competing scenarios influenced their post-decision moral reflection: some nurses and Tom's parents questioned again the decision to stop treatment, whereas the doctors stayed with it. In this case imagination contributed to social frictions.

\subsubsection{Imagining Living with Yourself}

'Could I live with myself if I decided/did this or that?' is a consideration that often plays a role in moral deliberation and reflection. ${ }^{12}$ Here the decision is taken, but when it is put

\footnotetext{
${ }^{12}$ It is worth noting that this consideration is often neglected in moral theory. An exception is Arendt (2003), who argued in Responsibility and Judgment that asking yourself if you could live with yourself after committing certain deeds is a criterion for judgment (p. 44). This is part of thinking, which she defined as being engaged in a "dialogue between me and myself" (p. 45). According to her, the voice of conscience is not felt as an obligation, but rather as an 'I can't': "I cannot do certain things, because having done them I shall no longer be able to live with myself'(p. 97).
} 
under pressure by a change in the situation, this kind of consideration actually did play a role in their reflection. The parents asked themselves how they could live with themselves after taking the decision of non-treatment assuming that their child has a chance. For them it is not clear anymore if they had taken the right decision. Whatever scenario they choose, it will give raise to feelings of moral guilt. It was only after Tom's death they knew the answer. Tom's father:

If we had said 'continue the treatment' maybe we could have kept him alive for three months more. Maybe three months more of suffering. And if we had decided to stop the treatment we would have been left with a feeling that maybe something could have happened and that we took away that chance from Tom. Whatever we decided, it would leave us with a feeling of doubt and guilt. Therefore we could not face the decision and we put it with the doctors. But since now we are certain of his death, we do sometimes wonder if his twin brother did not fare better. $\mathrm{He}$ at least died immediately after birth.

Of course whether or not there is a change, or how big this chance is, is under discussion in this case. But the point we want to make here is that asking the question regarding living with yourself is an imaginative exercise that aids moral reflection. The construction of a moral self-image plays a key role in the decision process. This takes place on an individual level as well as on the level of the collective NICU practice. We can ask ourselves: Do I want to be the kind of person that takes such decisions, and do we, as a unit, want to take this kind of decisions? Reflective practitioners will (and do) ask both questions, since they want to achieve harmony among and with themselves.

Note that we employ here a concept that is similar to empathy: imagining living with yourself is also an imaginative mental act but rather than taking the other as its aim or object, it returns to the self. We imagine ourselves rather than the other. This may be plain. But what sets our notion of self-image apart from many other, influential accounts ${ }^{13}$ may not be this link to empathy or our stress on the imaginative dimension of the self-to-self movement, but our understanding of the role of self-image in the decision-process. We do not merely decide and act on our past selves and predict what our decision will mean for our future selves. Surely, we imagine our future selves after the decision: an imaginative act enables us to predict in a forward-looking manner what our decision will mean for us. But we also decide and act, not just predict, on the basis of this forward-looking sense of who we are, and not on the basis of a backward-looking sense alone. If we use our imagination, we are no longer the prisoners of our past selves.

\section{Conclusion: Re-imagining the Practice of Intensive Care}

\subsection{The Moral Role of Imagination}

We started from pragmatist suggestions that imagination plays a key role in moral reasoning. To explore what this may mean in practice, we looked at moral reasoning in a neonatal intensive care unit. We examined three stages in a decision-making process: decision about starting treatment taken in the delivery room, decisions about continuation of treatment taken during the staff meeting, and reflection after the decision was taken. Our

\footnotetext{
${ }^{13}$ See for example Korsgaard's (1996) account in The Sources of Normativity or Velleman's (2006) Self to Self. 
analysis shows that imagination plays a role by projecting scenarios, by allowing people to put themselves in the place of others, by redefining and demarcating moral categories, by making possible improvisation and tuning, and by creating new action options. Imagination proved to be crucial in dealing with uncertainty in medical practice. It allows to fill the knowledge gap due to lack of data or lack of data that point in one direction. It also provides alternative options and solutions to practical problems. Finally, it provides an alternative form of reasoning and decision-making, or is at least to be considered as a key part of it. The outcome of imaginative acts support decisions by allowing people to project scenarios and assess situations. Imagination also assists people in reflection after a decision has been taken by helping them to think of themselves, by constructing their own moral identity. Thus, imagination aids personal moral integration and harmonization.

We conclude that imagination helps to bring about at least four kinds of integration in the moral decision-making process. First, it aids personal integration by helping people to think of themselves as moral beings, as the kind of being who needs to be able to live with itself. For example, a father wants to be able to live with himself after he has taken the decision (not) to let the child be born, and in this process of moral identity construction imagination plays a role as projection of future scenarios (he imagines his life with a handicapped child). Secondly, it assists social integration by aiding the conciliation of the people involved in the decision-making process and their diverging perspectives on the moral problem. Consider again the discussion in the staff meeting room about whether or not to start a discontinuation policy. Imagination helped the staff members to reach a consensus by creating a new action option. Note that this social integration supports longterm harmonization within the larger unit, including the construction of its moral identity. Thirdly, imagination aids temporal integration by facilitating the parties to transcend the present moment and connect past, present, and future. We discussed this use of imagination when we analyzed the role of projection in making a prognosis in the delivery room. The actors involved in the decision needed to connect the present condition of the child with projected future scenarios. Fourthly, imagination plays an important role in processes of epistemological integration by helping to combine the various forms of knowledge, experience needed to make a moral decision. For example, consider again the difficulty for medical staff to combine protocols, moral categories, case studies, ultrasound data, their personal experience, knowledge about the particular situation of the child, and other forms of knowledge to make a prognosis and a decision about further treatment. Imagination allowed them to align and harmonize these different forms in order to reach an adequate moral decision.

\subsection{Limits}

This account of epistemological integration as an imaginative enterprise shows that imaginative decision-making is not a licence to boundless action, absolute freedom, or fantasy. Rather, the use of imagination is constrained by knowledge and experience, and by the particularities of the situation. Moreover, apart from these epistemological and situational constraints, its resolving power is limited as well. Although imagination does play a role in moral decision-making and reflection, as we have shown above, it is not a magic, instant solution to moral problems and decisions. First, if there were anything that was a magic solution to moral problems, there would be no freedom of choice, and therefore no moral decision problem. Secondly, we have shown that imagination is only one of the elements that play a role in moral decision-making. Imagination itself does not provide the decision but is a device, an aid. But even that is not always the case. 
Imagination can be used in different ways that do not necessarily contribute to moral decision-making and reflection. For example, imagination may provide scenarios that are competing and as such may obstruct or complicate decision-making processes. In Tom's case the parents used their imagination to project another future for Tom - one in which he would survive - and this gave rise to doubt about the treatment policy and caused frictions between the doctors and nurses. Imagination itself is morally neutral, we can use it in ways that provide us with an inadequate perception of the moral problem, and as such imagination alone does not guarantee a morally adequate outcome of the decision-making process.

Can principles ensure such an outcome? Our analysis shows that it would be incorrect to see imagination and principles as mutually exclusive. Neither using principles nor using imagination is a sufficient condition of a morally right decision. Using imagination is not enough, as we argued above, and using imagination does not imply that principles, rules, and protocols become irrelevant. Rather, both were key elements in the decision-making processes we studied. They were combined and aligned imaginatively with other elements (forms of knowledge, experience, particular features of the situation and context). The moral adequacy of the decision depends on the alignment of many elements and is not sufficiently described in terms of the 'application' of moral principles or categories to a particular case or situation.

On the basis of this discussion about the limits of imagination we can ask further questions about the conditions for the use imagination. What are the limits, and how can we influence them? For example, what is the role of the NICU context and/or 'Evidence Based Culture' on imagination as a skill, and as an ability (imaginability)? We cannot answer these questions within the space of this paper. But if imagination does play a role in moral reasoning, these questions are highly relevant, since an answer to these questions can further increase our understanding of moral reasoning and assist intensive care actors in achieving a better moral self-understanding.

\subsection{Hope}

With our discussion we hope to contribute to the development of theoretical accounts of the role of imagination in moral decision-making, but we also hope that these insights about the role of imagination in NICU practice can assist the people involved in that practice to take up their role as reflective practitioners. In the end, it is up to them, as individuals and as a team, to re-imagine the practice of intensive care, and to continue to make their daily decisions with hope and imagination.

Acknowledgements We would like to thank Lieke van der Scheer (Maastricht), Jan Bransen (Nijmegen), and Rob Houtepen (Maastricht) for their comments on an earlier draft of this paper presented at the $G E E F$ research seminar (Health Sciences, Maastricht University). We thank the Faculty of Arts and Culture of Maastricht University for the Breedtestrategie-grant, which allowed the first author a little more research time to work on this paper. We also wish to thank all people that contributed to previous work on neonatal intensive care by the second author, from which this paper benefited enormously. Finally, we are grateful to the anonymous reviewers from Ethical Theory and Moral Practice for their helpful comments.

\section{References}

Anspach R (1993) Deciding who lives: faithful choices in the intensive-care nursery. University of California Press, Berkeley, CA

Arendt H (2003) In: Kohn J (ed) Responsibility and judgment. Schocken, New York 
Becker PT, Grunwald PC (2000) Contextual dynamics of ethical decision making in the NICU. J Perinat Neonatal Nurs 14(2):58-72

Burell D, Hauerwas S (1977) From system to story: an alternative pattern for rationality in ethics. In: Engelhardt HT, Callahan D (eds) The foundations of ethics and its relationship to science: knowledge, value, and belief, vol. 2. Hastings, New York

Coeckelbergh M (2006) Regulation or responsibility? Autonomy, moral imagination, and engineering. Sci Technol Human Values 31(3):1-24

Dancy J (1993) Moral reasons. Blackwell, Oxford

Dancy J (2004) Ethics without principles. Oxford University Press, Oxford

Eliasson AH, Howard RS, Torrington KG, Dillard TA, Phillips CY (1997) Do-not-resuscitate decisions in the medical ICU. Comparing physician and nurse opinions. Chest 111(4):1106-1111

Fesmire S (2003) John Dewey and moral imagination. Indiana University Press, Bloomington, IN

Frohock FM (1986) Special care: medical decisions at the beginning of life. University of Chicago Press, Chicago, IL

Holm S, Gjerøe P, Grode G, Hartling O, Ibsen K, Marcussen H (1996) Ethical reasoning in mixed nursephysician groups. J Med Ethics 22(3):168-173

Johnson M (1993) Moral imagination: implications of cognitive science for ethics. University of Chicago Press, Chicago, IL

Korsgaard KM (1996) The sources of normativity. Cambridge University Press, Cambridge, UK

Koselleck R (1985) Futures past: on the semantics of historical time. Translated by K. Tribe. In: McCarthy T (ed) Studies in contemporary German social thought. The MIT Press, Cambridge, MA

Mesman J (2002) Ervaren pioniers: Omgaan met twijfel in de intensive care voor pasgeborenen. Aksant, Maastricht

Nussbaum MC (2001) Upheavals of thought: the intelligence of emotions. Cambridge University Press, New York

Velleman JD (2006) The genesis of shame. Philos Public Aff 30(1) (Winter 2001):27-52. Reprinted in Self to Self. Cambridge: Cambridge University Press

Vermeulen E (2004) Dealing with doubt: making decisions in a neonatal ward in the Netherlands. Soc Sci Med 59:2071-2085

Werhane PH (1999) Moral imagination and management decision-making. Oxford University Press, New York

Williams B (1981) Moral luck. In: Moral luck. Philosophical papers: 1973-1980. Cambridge University Press, Cambridge, UK

Williams B (1985) Ethics and the limits of philosophy. Routledge, London [2006] 